\title{
Characterization of laser-electron interaction at the BESSY II femtoslicing source
}

\author{
K. Holldack, T. Kachel, S. Khan, ${ }^{*}$ R. Mitzner, ${ }^{\dagger}$ and T. Quast \\ BESSY, Albert-Einstein-Strasse 15, 12489 Berlin, Germany \\ (Received 20 January 2005; published 25 April 2005)
}

\begin{abstract}
The energy modulation of 1.7-GeV electrons by femtosecond laser pulses was studied at the BESSY II "femtoslicing" source, a facility commissioned in 2004 for the purpose of producing sub-100 fs x-ray pulses. As a test case for future seeded free-electron lasers, the laser-electron interaction was investigated as function of various laser and electron beam parameters using different experimental methods.
\end{abstract}

DOI: 10.1103/PhysRevSTAB.8.040704

PACS numbers: 41.60. $-\mathrm{m}, 42.65 . \mathrm{Re}$

\section{INTRODUCTION}

A laser pulse copropagating with an electron bunch in an undulator modulates the electron energy if the resonance condition

$$
\lambda_{L}=\frac{\lambda_{U}}{2 \gamma^{2}}\left(1+\frac{K^{2}}{2}\right)
$$

is fulfilled, i.e., if the laser wavelength $\lambda_{L}$ equals the wavelength of spontaneous undulator radiation. Here, $\lambda_{U}$ is the period length of the undulator, $K$ is its deflection parameter, and $\gamma$ is the Lorentz factor of the electrons. The oscillatory energy modulation has a period length equal to $\lambda_{L}$ and an envelope corresponding to the laser pulse shape enlarged by $\lambda_{L} N_{U}$, the distance by which electrons slip relative to the laser field over $N_{U}$ undulator periods.

Laser-induced energy modulation has a number of promising applications, among them seeding schemes for free-electron lasers (FELs) such as high-gain harmonic generation (HGHG) [1], enhanced self-amplified spontaneous emission (ESASE) [2] or sideband seeding [3]. It is also the basis of "femtoslicing," a method to produce subpicosecond synchrotron radiation pulses in storage rings $[4,5]$, and of schemes to generate subfemtosecond $\mathrm{x}$-ray pulses in FELs [6-8]. Another potential application is to generate coherent-light replica of electron bunches for diagnostics purposes [9].

At BESSY II, a third-generation synchrotron radiation source with a $1.7-\mathrm{GeV}$ electron storage ring, a femtoslicing facility to produce $\mathrm{x}$-ray pulses of sub-100 fs duration [full width at half maximum (FWHM)] was constructed and commissioned in 2004 [10]. Its purpose is to study ultrafast structural and magnetic phenomena in matter, and to gain hands-on experience in view of the proposed BESSY soft$\mathrm{x}$-ray FEL [11]. Here, femtoslicing serves as a test case for FEL seeding schemes, allowing to study the dependence of the energy modulation process on various parameters.

\footnotetext{
*Electronic address: shaukat.khan@bessy.de

${ }^{\dagger}$ Now at Universität Münster, Physikalisches Institut, Wilhelm-Klemm-Strasse 10, 48149 Münster, Germany.
}

\section{A. The principle of femtoslicing and its implementation at BESSY}

Femtoslicing is based on energy modulation of beam electrons by ultrashort laser pulses in an undulator, the "modulator." The off-energy electrons are then transversely displaced by dispersive elements in order to extract their synchrotron radiation emitted in a subsequent device, the "radiator," which may be a dipole magnet, a wiggler or an undulator. This radiation has essentially the time structure of the laser pulse and can be $10^{3}$ times shorter than conventional synchrotron radiation pulses.

At BESSY II, the modulator U139 (a planar wiggler with $\lambda_{U}=139 \mathrm{~mm}, N_{U}=10$ ) and the radiator UE56 (an elliptical undulator with $\lambda_{U}=56 \mathrm{~mm}, N_{U}=30$ ) are both placed in the same straight section to minimize lengthening of the electron distribution between them. A dipole magnet with a bending angle of $112 \mathrm{mrad}$ following the modulator displaces the energy-modulated electrons by $1 \mathrm{mrad}$ such that radiation from the bunch core does not overlap with the short-pulse component and can be blocked by an aperture. This angular separation scheme avoids imaging elements, which could cause an intolerably large background due to nonspecular scattering [12]. A liquid-nitrogen-cooled Ti:sapphire laser system [13] provides pulses at a wavelength of $\lambda_{L}=800 \mathrm{~nm}$ with a pulse energy up to $2.8 \mathrm{~mJ}$ at $1 \mathrm{kHz}$ (alternatively $1.8 \mathrm{~mJ}$ at $2 \mathrm{kHz}$ ) and a pulse duration of $\geq 30 \mathrm{fs}$ (FWHM). The laser pulses are focused by a two-lens telescope. After passing the telescope, the laser pulses can be sent back to the laser hutch for diagnostics purposes, where the transverse beam shape is observed by a CCD camera and the pulse duration is measured by standard autocorrelation techniques. Further details are given in [14]. Figure 1 shows part of the BESSY II floor plan, where the features related to the femtoslicing facility are highlighted.

\section{B. Relevant parameters}

The electron beam parameters of interest are the electron energy or Lorentz factor, energy spread, bunch length, and the transverse beam size given by emittance, beta functions and dispersion. 


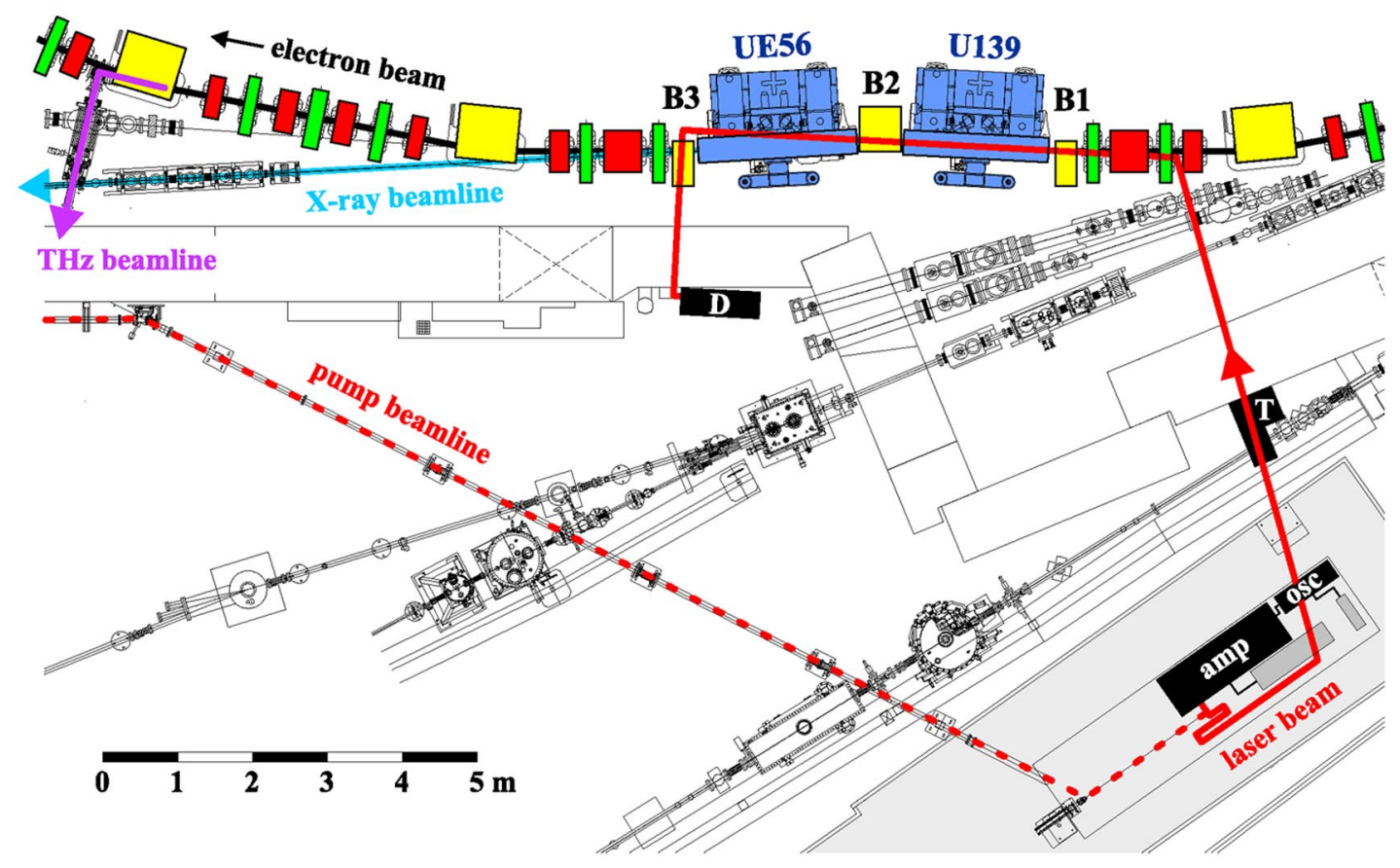

FIG. 1. (Color) BESSY II floor plan showing a part of the electron storage ring with dipole (yellow), quadrupole (red), and sextupole magnets (green). A closed orbit bump (B1-B2-B3) encloses the modulator (U139) and the radiator (UE56). A hutch (gray) outside the ring tunnel contains a Ti:sapphire oscillator (osc) and amplifier (amp). The laser beam (solid red line) is focused by a telescope (T) and ends at a diagnostics station (D), while pump pulses (dotted red line) are directed to the experiment at the end of the x-ray beam line (blue). The energy modulation is monitored using THz radiation (magenta).

The relevant laser properties are wavelength, bandwidth, longitudinal and transverse chirp, polarization, pulse energy and duration, as well as the transverse beam size and divergence, which are given by the wavelength and the quality parameter $M^{2}$, and are subject to optical distortions such as astigmatism.

The undulator is specified by its period length, the number of periods, and to first order by the deflection parameter $K$. For an undulator matching the resonance condition of Eq. (1) for $\lambda_{L}=800 \mathrm{~nm}$, higher harmonics of the magnetic field are also relevant.

Finally, the interaction efficiency depends critically on the overlap of laser pulse and electron bunch in all phase space coordinates, i.e., horizontal and vertical position and angle, timing as well as spectral overlap of laser and spontaneous undulator radiation. Apart from a static mismatch, there may be oscillations in any coordinate caused by longitudinal or transverse instabilities of the electron beam, by mechanical vibrations, or by insufficient pointing stability or temporal jitter of the laser.

\section{PREDICTION OF ENERGY MODULATION}

A suitable figure of merit is the modulation amplitude, i.e., the maximum energy transfer between an electron and the laser field. For femtoslicing, the modulation amplitude determines the maximum transverse displacement. For FEL seeding, it is related to the bunching factor.
Another useful quantity is the number of electrons exceeding a certain energy offset. When designing the BESSY femtoslicing facility, for example, electrons with $\Delta E / E$ exceeding $0.7 \%$ were assumed to be relevant for separable short-pulse radiation.

\section{A. Analytical estimate}

The amplitude $\Delta E$ of the oscillatory energy modulation is approximately given by [4]

$$
(\Delta E)^{2}=4 \pi \alpha A_{L} E_{L} \frac{K^{2} / 2}{1+K^{2} / 2} \frac{\Delta \omega_{L}}{\Delta \omega_{U}},
$$

where $\alpha$ is the fine structure constant, $A_{L}$ is the pulse energy, $E_{L}$ is the photon energy, and $K$ is the deflection parameter. The bandwidth ratio $\Delta \omega_{L} / \Delta \omega_{U}$ of laser and undulator radiation can be approximated by $N_{U} / N_{L}$, the ratio of undulator periods and optical cycles in the laser pulse. This ratio is limited to unity, since there is no further gain once the electron slippage $N_{U} \lambda_{L}$ exceeds the laser pulse length $N_{L} \lambda_{L}$. There are corrections to Eq. (2) for matching laser and undulator spectra, and to account for the finite electron beam size [12], but the dependence on other parameters has to be studied by numerical simulations or experimentally. 


\section{B. Numerical simulation}

Prior to the construction of the femtoslicing facility at BESSY II, numerical simulations were employed to determine the laser requirements (such as pulse energy, pulse duration, and $M^{2}$ ), to study the effect of imperfect electronlaser overlap and other deviations from an ideal situation, and to verify the feasibility of the angular separation scheme. The computation of a realistic distribution of energy-modulated electrons allows one to predict the properties of the resulting $\mathrm{x}$-ray pulses (pulse duration, photon rate, background, spatial distribution, and spectral characteristics), as well as the further evolution of the longitudinal bunch shape, which gives rise to coherent synchrotron radiation (CSR) in the $\mathrm{THz}$ regime.

The laser-induced energy deviation $\Delta E$ is computed by numerically integrating the product of the force exerted by the electric field $\mathcal{E}$ and the transverse electron displacement $x^{\prime} \equiv d x / d s$ per infinitesimal step $d s$ over the length $L=$ $N_{U} \lambda_{U}$ of the modulator for an ensemble of randomly generated "macroelectrons":

$$
\Delta E=-e \int_{-L / 2}^{L / 2} x^{\prime}(s) \mathcal{E}(x, y, z) d s .
$$

The electric field of the laser pulse is given by [15]

$$
\mathcal{E}(x, y, z)=\sqrt{2 \rho(x, y, z) / \varepsilon_{0}} \sin \left[2 \pi z / \lambda_{L}-\varphi_{G}\left(s_{L}\right)\right] .
$$

Here, $(x, y, z)$ is the time-dependent electron position relative to the laser pulse center, $\rho(x, y, z)$ is the energy density, $\varepsilon_{0}$ is the permittivity of free space, $s_{L}$ is the laser pulse position relative to the modulator center at $s=0$, and

$$
\varphi_{G}\left(s_{L}\right)=\tan ^{-1}\left(\frac{s_{L} \lambda_{L}}{4 \pi \sigma_{x, y}^{2}(0)}\right)
$$

is the Guoy phase shift with a beam waist at $s=0$. The laser pulse is assumed to be Gaussian in the longitudinal and transverse coordinates. Its horizontal and vertical rms size is a function of $s_{L}$ and depends on the respective quality parameter $M_{x, y}^{2}$ :

$$
\sigma_{x, y}\left(s_{L}\right)=\sqrt{\sigma_{x, y}^{2}(0)+\left(\frac{M_{x, y}^{2} \lambda_{L}}{4 \pi \sigma_{x, y}(0)}\right)^{2} s_{L}^{2}} .
$$

The numerical model is easily adapted to describe elliptical beams, astigmatism and other aberrations, phase modulation, and any kind of mismatch between laser pulse and electron bunch. Modifications of the laser field in this low-gain process are assumed to be small and are not included in the model.

The result of the simulation is the amount of energy $\Delta E$ acquired by each macroelectron in the interaction process. The end point of their distribution along the $\Delta E$ axis is the modulation amplitude. In order to obtain the intended modulation amplitude of $\Delta E / E=0.01$ for a laser pulse duration of, e.g., 45 fs (FWHM) and $M_{x, y}^{2} \leq 1.5$, the simulation suggests a pulse energy of $1.5 \mathrm{~mJ}$.

\section{EXPERIMENTAL METHODS}

Laser-induced energy modulation of electrons leads to longitudinal and transverse changes of the electron distribution, and to modifications of the laser spectrum. These effects can, in turn, be employed to characterize the modulation process.

\section{A. Longitudinal electron distribution}

Energy-modulated electrons change their longitudinal position $z$ according to

$$
z\left(s_{1}\right)-z\left(s_{0}\right)=R_{51} x\left(s_{0}\right)+R_{52} x^{\prime}\left(s_{0}\right)+R_{56} \Delta E / E
$$

as they propagate through the magnetic lattice from position $s_{0}$ to $s_{1}$ [16]. For the case of BESSY II, the coefficients $R_{51}$ and $R_{52}$ are of particular relevance since the energy change takes place in a region of nonzero dispersion and a betatron oscillation is excited.

\section{Microbunching}

Energy-dependent differences of the electron path length below $\lambda_{L} / 2$ lead to the formation of microbunches, a periodic density modulation that causes CSR at wavelengths above $\approx 1 / 5$ of $\lambda_{L}$. The fact that this radiation is further enhanced at integer fractions of the laser wavelength (higher harmonics), is explicitly exploited in the HGHG scheme. At the BESSY femtoslicing facility, microbunching is predicted to occur at certain positions along the electron beam trajectory at which, however, the radiation cannot easily be extracted and it was not yet investigated.

\section{Coherent $\mathrm{THz}$ radiation}

For path length differences exceeding the laser pulse length, the laser-electron overlap region is depleted and a dip occurs in the longitudinal electron distribution. Its size and shape depend on the laser pulse duration, on the acquired energy modulation, and on the properties of the magnetic lattice entering Eq. (7). A short dip of the order of $0.1 \mathrm{~mm}$ gives rise to CSR in the $\mathrm{THz}$ regime, which can be used as a very sensitive and reliable on-line diagnostics tool. At BESSY II, a dedicated THz beam line was constructed at a bending magnet $11 \mathrm{~m}$ downstream of the modulator [17].

Generally, the radiation power at a given wave number $1 / \lambda$ emitted by an electron distribution $\varrho(z)$ is [16]

$$
P(1 / \lambda)=N p_{1 / \lambda}+f_{1 / \lambda} N(N-1) p_{1 / \lambda},
$$

where $p_{1 / \lambda}$ is the power emitted by a single electron, $N$ is the number of electrons, and $f_{1 / \lambda}$ is a form factor linked to 
the electron distribution via Fourier transform:

$$
f_{1 / \lambda}=\left|\int e^{i 2 \pi z / \lambda} \varrho(z) d z\right|^{2}
$$

At BESSY II, THz radiation measured with an InSb bolometer is routinely employed to detect laser-induced energy modulation and to optimize the spatial, temporal, and spectral laser-electron overlap. Using a lock-in amplifier, the detector output is usually sampled at the 27th harmonic of the laser repetition frequency of $998 \mathrm{~Hz}$. Its dependence on several critical parameters is illustrated by Figs. 2-4.

Figure 2 shows the $\mathrm{THz}$ signal amplitude as a function of the resonant wavelength of the modulator. Tuning the gap off-resonance reduces the modulation amplitude because electrons do not experience the maximum field over all undulator periods. The optimum modulator wavelength is detuned from the central laser wavelength by $\approx 10 \mathrm{~nm}$ as a consequence of the Guoy phase shift [18].

In Fig. 3, the THz signal is shown as a function of the laser pulse delay relative to the rf phase that determines the electron bunch position. In contrast to the modulator gap variation discussed above, the number of participating electrons changes, while the modulation amplitude re-
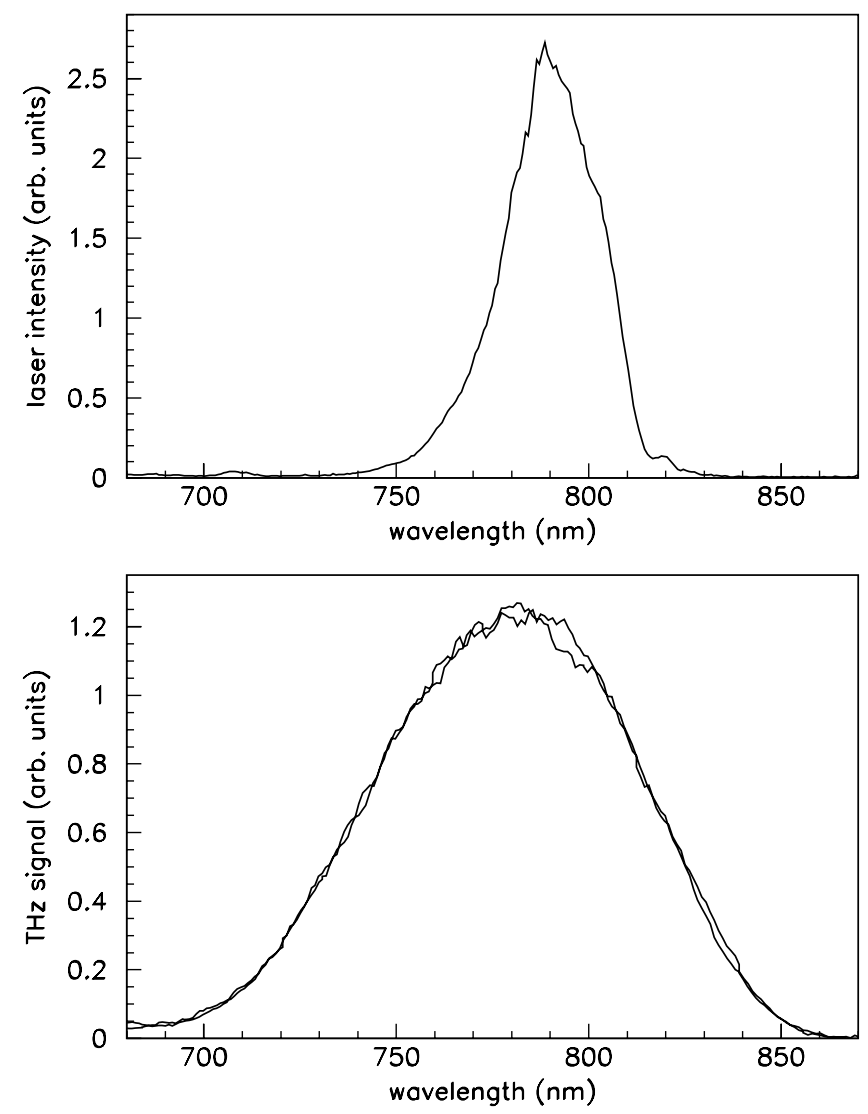

FIG. 2. Laser spectrum (top) and intensity of $\mathrm{THz}$ radiation from energy-modulated electrons for several scans of the resonant modulator wavelength (bottom).

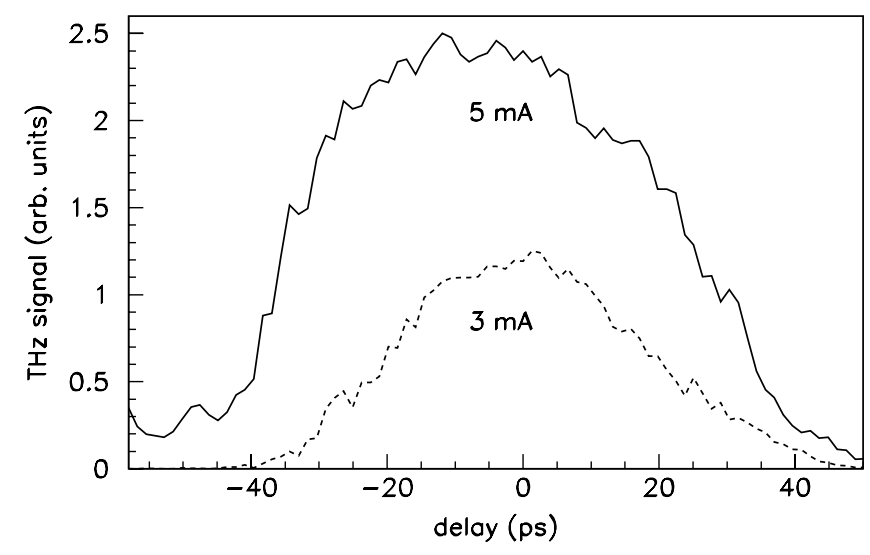

FIG. 3. THz radiation intensity from energy-modulated electrons under variation of the laser pulse delay for an electron bunch current of 3 and $5 \mathrm{~mA}$.

mains the same. The square root of the $\mathrm{THz}$ signal is proportional to the electron density in the overlap region and allows one to probe the bunch shape with a resolution corresponding to the laser pulse length. Evidently, the shape, length, and centroid position of the bunch depends on its current, for which wake field effects (potential-well distortion, longitudinal mode-coupling instability [19]) as well as CSR-driven forces [20] may be responsible. The results obtained here confirm previous bunch length measurements using a streak camera [21].

Figure 4 shows the dependence of the $\mathrm{THz}$ signal on the transverse overlap of laser and electrons. Here, the electron beam was displaced horizontally while monitoring laser
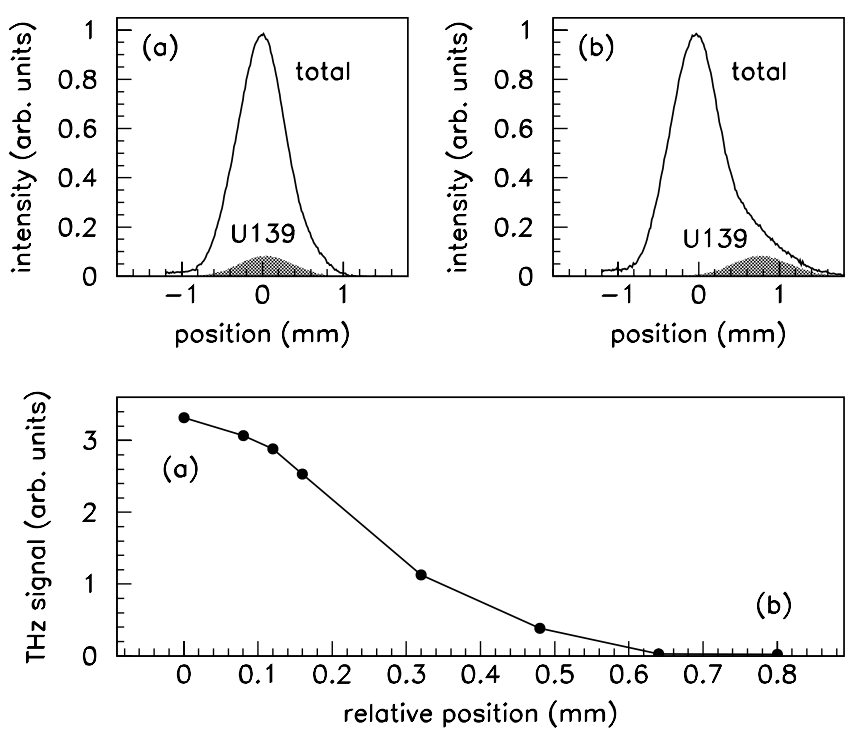

FIG. 4. Horizontal profile of synchrotron radiation from the U139 modulator with and without laser pulses as observed with a CCD camera (a) at optimum overlap and (b) displaced by 0.8 $\mathrm{mm}$. The bottom part of the figure shows the $\mathrm{THz}$ radiation intensity as a function of the displacement. 
and spontaneous radiation from the modulator using a telescope with a CCD camera. Contrary to the longitudinal case, the laser pulse cannot be considered as a small probe and a displacement changes the modulation amplitude as well as the number of participating electrons.

These examples show that the signal from $\mathrm{THz}$ radiation integrated over time and wave number is well suited to detect and optimize the energy modulation process, but quantitative conclusions regarding the energy modulation are not easily drawn. Additional information may be obtained from the radiation spectrum and by time-resolved measurements. Work in this direction is in progress.

\section{B. Transverse electron distribution}

The energy modulation $\Delta E / E$ at a position with optical functions $\tilde{\beta}_{x}, \tilde{\alpha}_{x}, \tilde{\gamma}_{x}$, dispersion $\tilde{D}$ and $\tilde{D}^{\prime}=d \tilde{D} / d s$ excites a horizontal betatron oscillation relative to the dispersive orbit $x_{D}(s)=(\Delta E / E) D(s)$. The resulting CourantSnyder invariant is given by [16]

$$
\varepsilon_{x}=\left(\frac{\Delta E}{E}\right)^{2}\left(\tilde{\gamma}_{x} \tilde{D}^{2}+2 \tilde{\alpha}_{x} \tilde{D} \tilde{D}^{\prime}+\beta_{x} \tilde{D}^{\prime 2}\right) .
$$

\section{Scraper measurements}

With a scraper positioned at a distance $\Delta x$ from the beam center, electrons contribute to the loss rate (i.e., the inverse beam lifetime) if

$$
\Delta x \leq \sqrt{\varepsilon_{x} \beta_{x}\left(s_{1}\right)},
$$

where $\beta_{x}\left(s_{1}\right)$ is the horizontal beta function at the location of the scraper and $D\left(s_{1}\right)$ is zero. The top part of Fig. 5 shows the beam lifetime as a function of time, derived from a current measurement using a Si photodiode (described in [22]), while the scraper is moved towards the beam and the laser is blocked periodically. The bottom part shows the loss rate, i.e., the inverse lifetime as a function of the scraper position relative to the beam center deduced from the position at which the quantum lifetime [16] becomes dominant and the beam is lost. The solid line is derived simulating the energy modulation under variation of the laser pulse energy assuming perfect laser-electron overlap and using measured longitudinal and transverse laser pulse dimensions. In all experiments, the actual laser power measured with a power meter is significantly higher (by a factor 1.5-2) than that found from simulations. This may be due to insufficient overlap, power losses on the way to the interaction region, or other effects that reduce the efficiency of the laser-electron interaction such as optical aberrations.

\section{Synchrotron radiation distribution}

Another transverse effect of energy modulation is, according to the actual purpose of femtoslicing, the appearance of additional photons at the fringes of the angular
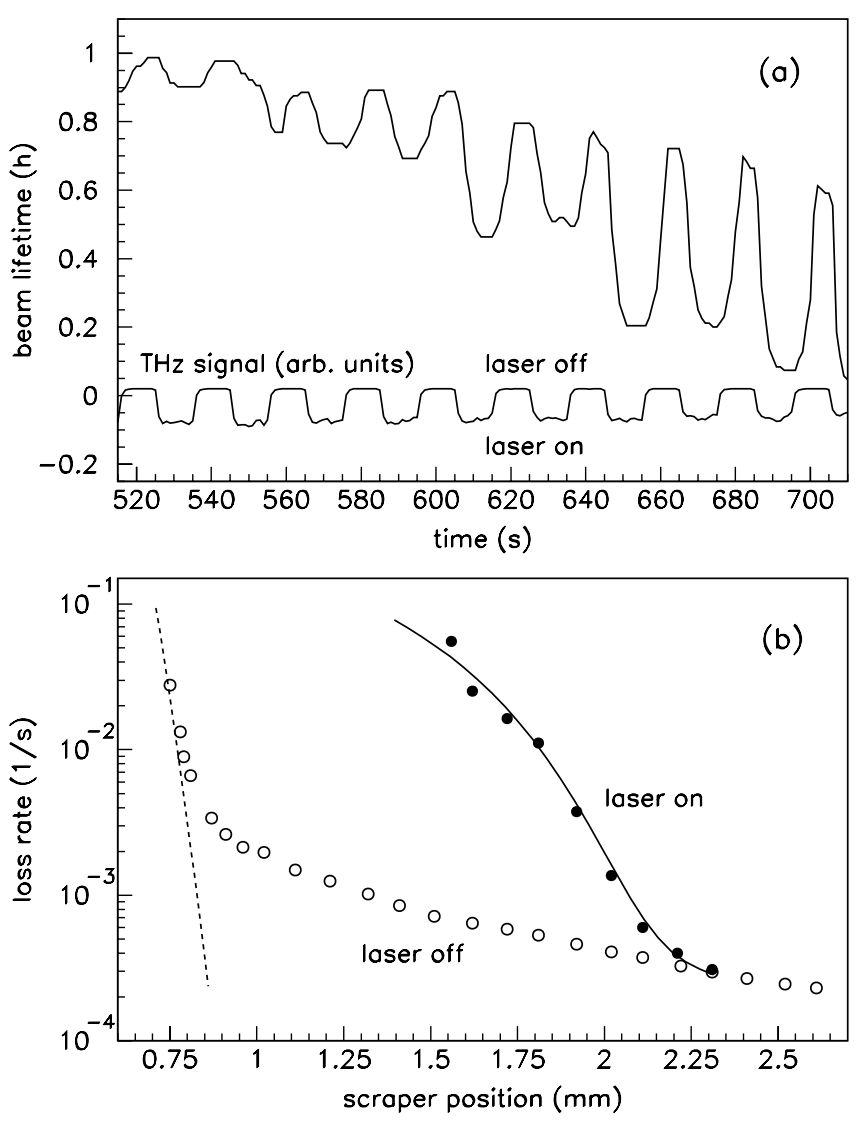

FIG. 5. (a) Beam lifetime and $\mathrm{THz}$ radiation intensity as function of time, while a scraper is moved towards the beam and the laser is blocked periodically. (b) Loss rate versus scraper position, where the dashed line indicates the quantum lifetime limit and the solid line is the result of a simulation using measured laser pulse dimensions and varying the laser pulse energy.

synchrotron radiation distribution. As a first indication, modifications of the angular distribution can be detected in time-integrated measurements, even though the laser repetition rate is 1250 times lower than the single-bunch rate. This is due to the fact that the synchrotron and betatron oscillations, once excited by the energy modulation, persist over $\sim 10^{4}$ turns according to the longitudinal and transverse damping times, respectively. Consequently, radiation from the same electron is detected repeatedly, and the repetition factor depends on the energy offset of that electron and on the observation angle.

The top part of Fig. 6 shows the photocurrent from a GaAs diode behind the exit slit of a plane grating monochromator [23]. The angle was changed by moving an aperture in the front end across the radiation distribution of the UE56 radiator. As the angle increases, the fraction of radiation from energy-modulated electrons should increase. On the other hand, the probability of detecting them repeatedly decreases, resulting in an enhancement of the order of $10 \%$ at best. 

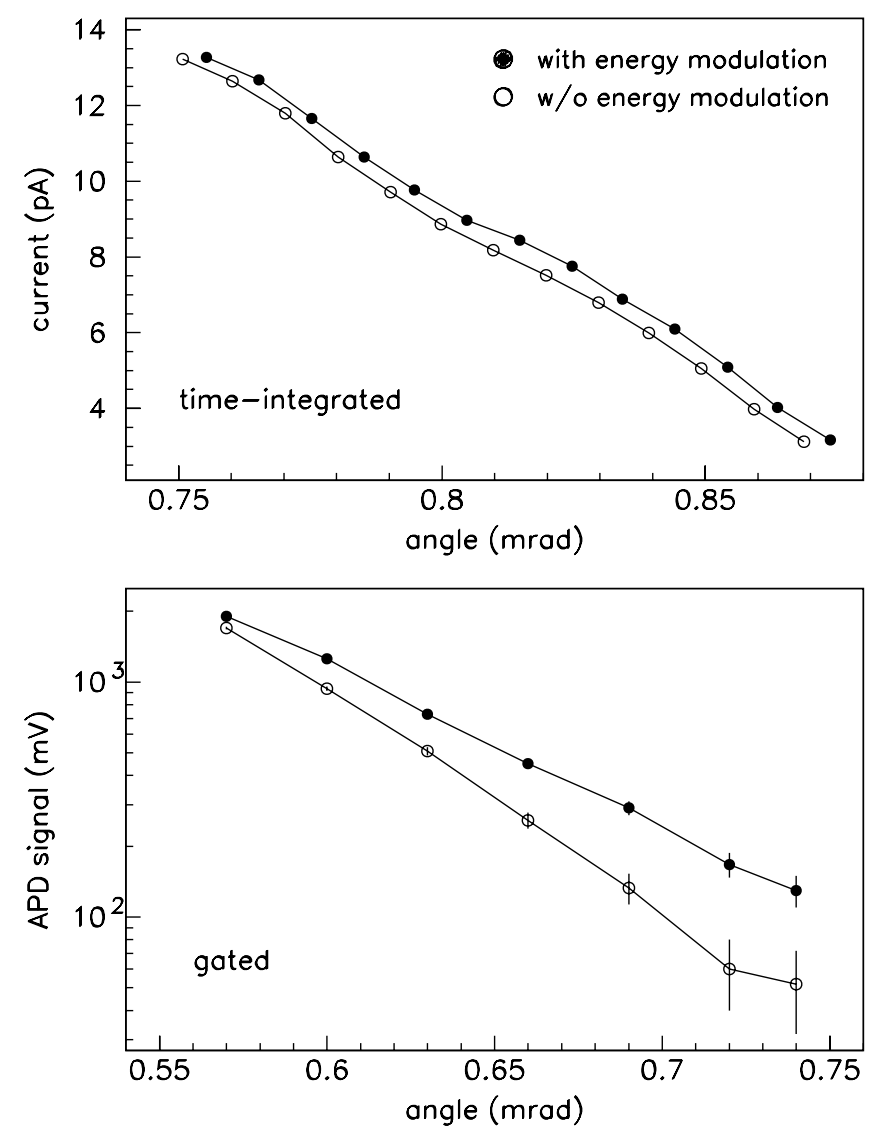

FIG. 6. Angular distribution of the synchrotron radiation intensity at $708 \mathrm{eV}$ from a time-integrated measurement using a GaAs diode (top) and from an avalanche photodiode (APD) gated synchronously with the laser pulse (bottom).

The fraction of short-pulse radiation from energymodulated electrons is determined by a time-resolved measurement of the pulse amplitude from a fast avalanche photodiode (APD). A digital oscilloscope of $2 \mathrm{GHz}$ bandwidth, triggered by a signal from the Ti:sapphire amplifier, was used to distinguish the APD signal of the interacting bunch from the radiation of adjacent bunches. After passing the monochromator, the number of photons is proportional to the detected pulse height, which is averaged over several $10^{3}$ oscilloscope sweeps. Reducing the interaction rate for each bunch to $200 \mathrm{~Hz}$ by alternately selecting five different bunches eliminates the radiation background from electrons excited at previous interactions. The bottom part of Fig. 6 shows the time-resolved measurement of radiation from the UE56 radiator as a function of angle, obtained by changing the angle of the electron beam axis while keeping the front end aperture fixed. The difference between the measurements with and without energy modulation is the signal from energy-modulated electrons. Above $0.7 \mathrm{mrad}$, the signal-to-background ratio clearly exceeds $1: 1$. This first measurement was limited by electronic noise to signals above $50 \mathrm{mV}$, and measurements with a better signal-to-background ratio are under way.
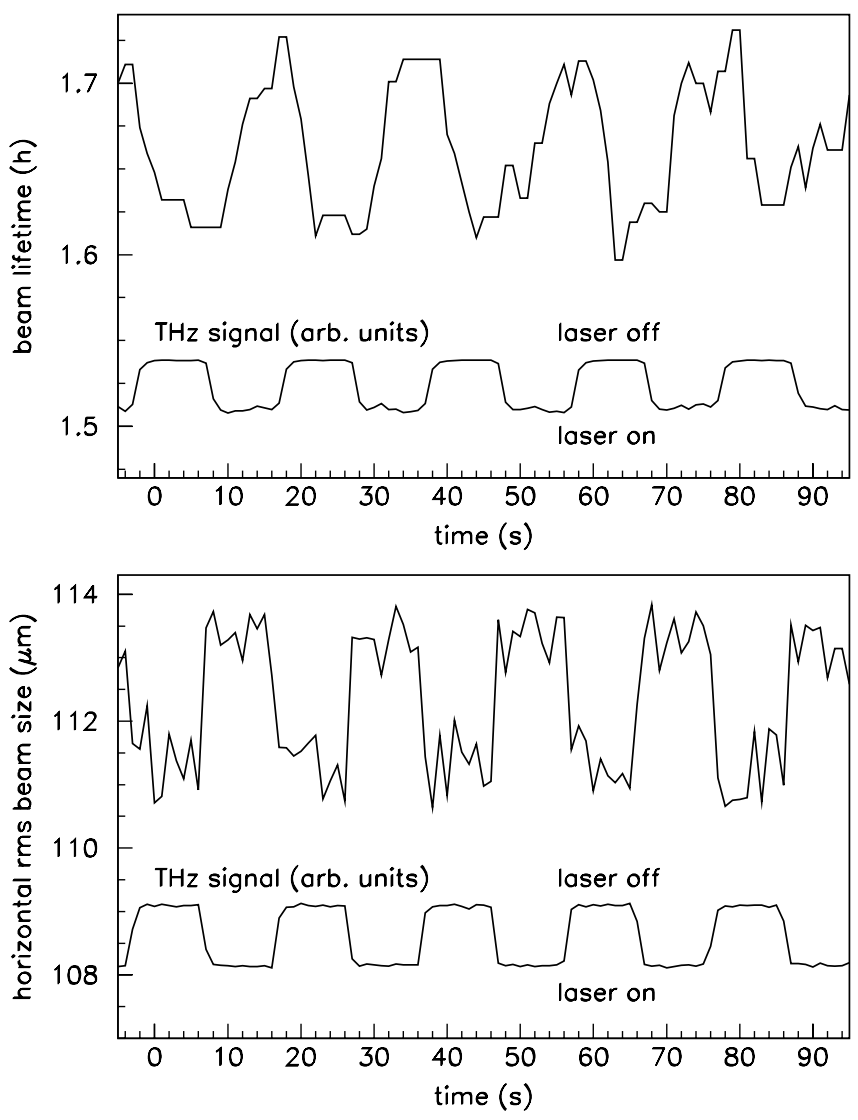

FIG. 7. Single-bunch lifetime (top) and horizontal rms beam size (bottom) as function of time, while the laser is blocked periodically as indicated by the $\mathrm{THz}$ signal.

\section{Other effects on the electron beam}

With a single bunch in the BESSY II storage ring, there are further observations related to longitudinal and transverse changes of the electron distribution due to energy modulation:

(i) Radiation from energy-modulated electrons can be directly observed by a CCD camera behind a pinhole as an elliptical halo surrounding the core beam. The rms size deduced from the beam image is enlarged by $1-2 \%$, as shown in the bottom part of Fig. 7.

(ii) Energy modulation reduces the electron density in phase space and thus increases the lifetime of a single bunch, which is dominated by electronelectron scattering (Touschek effect). In the example shown in Fig. 7, the lifetime increases by $6 \%$.

(iii) The reduction of the electron density influences the intensity of synchrotron radiation at some of the user experiments on the level of $1 \%$.

Again, these effects are a consequence of the persistence of the synchrotron and betatron oscillations excited by energy modulation in a dispersive region. With multiple stored bunches, they are suppressed by the ratio of the total 
beam current to the current of the interacting bunch. As a practical consequence, parasitic experiments with energy modulation during user beamtime is restricted to multibunch operation.

Both, the transverse and longitudinal distribution of energy-modulated electrons can be mapped by crosscorrelating visible radiation from a dipole magnet with a delayed laser pulse while moving a slit across the beam image $[5,12]$. This technique was not pursued at BESSY, because the UE56 radiator does not produce visible light. The longitudinal electron distribution can also be obtained, albeit less directly, from the spectrum of $\mathrm{THz}$ radiation using a model for the evolution of the longitudinal electron distribution.

\section{Effect on the laser pulse}

Depending on the setting of the deflection parameter $K$ of the modulator, there is a net energy transfer from the electrons to the laser field or vice versa, corresponding to the gain of a single-pass FEL or an inverse FEL, respectively. This gain can be observed in the laser spectrum as a difference of the order of $0.5 \%$ between the light intensity above and below the central wavelength [5,12]. Again, this method was not employed at BESSY, mainly due to the availability of the $\mathrm{THz}$ diagnostics system.

\section{CONCLUSIONS}

There are several signatures of the interaction between a laser pulse and electrons in a storage ring, resulting in a periodic modulation of their energy. Some effects merely hint the occurrence of this interaction, others allow to draw quantitative conclusions. At the BESSY II femtoslicing facility, the best everyday tool to detect and optimize laser-induced energy modulation is the detection of coherent $\mathrm{THz}$ radiation. A quantitative study of the modulation relies presently on probing the transverse electron distribution with a scraper and on measuring the angular distribution of monochromatized synchrotron radiation in the $\mathrm{x}$-ray beam line.

With suitable adaptions, all time-resolved methods should be applicable to linear accelerators as well, allowing - for example - to monitor the energy modulation process in a seeded FEL.

\section{ACKNOWLEDGMENTS}

We would like to express our gratitude to all BESSY colleagues contributing to the femtoslicing project, among them J. Bahrdt, H. A. Dürr, V. Dürr, W. B. Peatman, M. Scheer, F. Senf, G. Wüstefeld, and many others. Helpful discussions with F. Noack, I. Will (MBI, Berlin), A. Zholents (LBNL, Berkeley), G. Ingold, A. Streun (SLS, Villigen) and hardware support by T. Lohse (HU, Berlin) are gratefully acknowledged. This work was funded by the
Bundesministerium für Bildung und Forschung and by the Land Berlin.

[1] L. H. Yu, Phys. Rev. A 44, 5178 (1991).

[2] A. A. Zholents, W. M. Fawley, P. Emma, Z. Huang, G. Stupakov, and S. Reiche, in Proceedings of the 26th International Free Electron Conference, Trieste, 2004, http://www.jacow.org, p. 582.

[3] W. Brefeld et al., Nucl. Instrum. Methods Phys. Res., Sect. A 483, 62 (2002).

[4] A. A. Zholents and M. S. Zoloterev, Phys. Rev. Lett. 76, 912 (1996).

[5] R. W. Schoenlein et al., Science 287, 2237 (2000).

[6] A. A. Zholents and W. M. Fawley, Phys. Rev. Lett. 92, 224801 (2004).

[7] E. L. Saldin, E. A. Schneidmiller, and M. V. Yurkov, Opt. Commun. 237, 153 (2004).

[8] E. L. Saldin, E. A. Schneidmiller, and M. V. Yurkov, Opt. Commun. 239, 161 (2004).

[9] E. L. Saldin, E. A. Schneidmiller, and M.V. Yurkov, DESY Report No. 04-126, 2004.

[10] S. Khan et al., in Proceedings of the European Particle Accelerator Conference, Lucerne, 2004, http://www. jacow.org, p. 2287.

[11] M. Abo-Bakr et al., in The BESSY Soft X-Ray Free Electron Laser, Technical Design Report, edited by D. Krämer, E. Jaeschke, and W. Eberhardt (BESSY, Berlin, 2004).

[12] R. W. Schoenlein et al., Appl. Phys. B 71, 1 (2000).

[13] Kapteyn-Murnane Laboratories Inc. MTS oscillator and HAP-AMP amplifier, Coherent Inc. Verdi V5, Quantronix 527DQE-S.

[14] S. Khan et al., in Proceedings of the Particle Accelerator Conference, Portland, 2003, http://www.jacow.org, p. 836.

[15] A.E. Siegman, Lasers (University Science Books, Sausalito, 1986).

[16] H. Wiedemann, Particle Accelerator Physics (Springer, Berlin, 1993).

[17] K. Holldack, S. Khan, R. Mitzner, T. Quast, and G. Wüstefeld, in Proceedings of the European Particle Accelerator Conference, Lucerne, 2004, http://www. jacow.org, p. 2284.

[18] A. Amir and Y. Greenzweig, Phys. Rev. A 34, 4809 (1986).

[19] A. W. Chao, Physics of Collective Beam Instabilities in High Energy Accelerators (John Wiley \& Sons, New York, 1993).

[20] F. Sannibale et al., Phys. Rev. Lett. 93, 094801 (2004).

[21] P. Kuske, M. Abo-Bakr, W. Anders, and G. Wüstefeld, in Proceedings of the Particle Accelerator Conference, Portland, 2003, http://www.jacow.org, p. 3020.

[22] R. Thornagel, R. Klein, and G. Ulm, Metrologia 38, 385 (2001).

[23] M.R. Weiss et al., Nucl. Instrum. Methods Phys. Res., Sect. A 467, 449 (2001). 